\title{
Lung Ultrasound for Detection of Pulmonary Complications in Critically III Obstetric Patients in a Resource-Limited Setting
}

Luigi Pisani, ${ }^{1,2 \star} \dagger$ Anna De Nicolo, ${ }^{3} \dagger$ Marcella Schiavone,${ }^{4} \dagger$ Adetunji O. Adeniji, ${ }^{5}$ Angela De Palma, ${ }^{4}$ Francesco Di Gennaro, ${ }^{6}$ Edward Ejiro Emuveyan, ${ }^{5}$ Salvatore Grasso, ${ }^{3}$ Patricia C. Henwood, ${ }^{7}$ Alimamy P. Koroma, ${ }^{5}$ Stije Leopold, ${ }^{2,8}$ Claudia Marotta, ${ }^{6}$ Giuseppe Marulli, ${ }^{4}$ Giovanni Putoto, ${ }^{6}$ Enzo Pisani, ${ }^{5}$ James Russel, ${ }^{9}$ Ary Serpa Neto, ${ }^{10}$ Arjen M. Dondorp, ${ }^{2,11}$ Eva Hanciles, ${ }^{12}$ Michael M. Koroma, ${ }^{5}$ and Marcus J. Schultz ${ }^{1,2,11,13}$

${ }^{1}$ Department of Intensive Care, Amsterdam University Medical Centers-Location AMC, Amsterdam, The Netherlands; ${ }^{2}$ Mahidol-Oxford Tropical Medicine Research Unit (MORU), Mahidol University, Bangkok, Thailand; ${ }^{3}$ Intensive Care Unit, Department of Emergency and Organ Transplantation (DETO), University of Bari, Bari, Italy; ${ }^{4}$ Thoracic Surgery Unit, Department of Emergency and Organ Transplantation (DETO), University of Bari, Bari, Italy; ${ }^{5}$ Princess Christian Maternity Hospital, Freetown, Sierra Leone; ${ }^{6}$ Section of Operational Research, Doctors with Africa-Cuamm, Padova, Italy; ${ }^{7}$ Department of Emergency Medicine, Thomas Jefferson University Hospitals, Thomas Jefferson University, Philadelphia, Pennsylvania; ${ }^{8}$ Department of Internal Medicine, Amsterdam University Medical Centers - Location AMC, Amsterdam, The Netherlands; ${ }^{9}$ Department of Cardiology, Connaught Hospital, University of Sierra Leone, Freetown, Sierra Leone; ${ }^{10}$ Department of Critical Care Medicine, Hospital Israelita Albert Einstein, São Paulo, Brazil; ${ }^{11}$ Nuffield Department of Medicine, University of Oxford, Oxford, United Kingdom;

${ }^{12}$ Department of Anesthesia and Intensive Care, Connaught Hospital, University of Sierra Leone, Freetown, Sierra Leone; ${ }^{13}$ Laboratory of Experimental Intensive Care and Anesthesiology (L.E.I.C.A), Amsterdam University Medical Centers - Location AMC, Amsterdam, The Netherlands

\begin{abstract}
Critically ill parturients have an increased risk of developing pulmonary complications. Lung ultrasound (LUS) could be effective in addressing the cause of respiratory distress in resource-limited settings with high maternal mortality. We aimed to determine the frequency, timing of appearance, and type of pulmonary complications in critically ill parturients in an obstetric unit in Sierra Leone. In this prospective observational study, LUS examinations were performed on admission, after 24 and 48 hours, and in case of respiratory deterioration. Primary endpoint was the proportion of parturients with one or more pulmonary complications, stratified for the presence of respiratory distress. Secondary endpoints included timing and types of complications, and their association with "poor outcome," defined as a composite of transfer for escalation of care or death. Of 166 patients enrolled, 35 patients (21\% [95\% Cl: 15-28]) had one or more pulmonary complications, the majority diagnosed on admission. Acute respiratory distress syndrome (period prevalence $4 \%$ ) and hydrostatic pulmonary edema $(4 \%)$ were only observed in patients with respiratory distress. Pneumonia (2\%), atelectasis (10\%), and pleural effusion $(7 \%)$ were present, irrespective of respiratory distress. When ultrasound excluded pulmonary complications, respiratory distress was related to anemia or metabolic acidosis. Pulmonary complications were associated with an increased risk of poor outcome (odds ratio: $5.0 ; 95 \% \mathrm{Cl}$ : 1.7-14.6; $P=0.003$ ). In critically ill parturients in a resource-limited obstetric unit, LUS contributed to address the cause of respiratory distress by identifying or excluding pulmonary complications. These were associated with a poor outcome.
\end{abstract}

\section{INTRODUCTION}

Maternal mortality in Sierra Leone is among the highest in the world, with 1,360 maternal deaths $/ 100,000$ babies born alive. ${ }^{1}$ Major direct obstetric complications are an important cause of increased mortality. ${ }^{2}$ The three leading causes of mortality and morbidity during pregnancy are peripartum hemorrhage, sepsis, and preeclampsia ${ }^{3,4}$-each of these directly or indirectly predispose for pulmonary complications. ${ }^{3}$ Pulmonary complications requiring transitory or intense critical care treatment may arise before, during, and even after the primary obstetric disease is resolved. Among obstetric patients in Sierra Leone, additional risk factors for developing pulmonary complications include pregnancy-associated cardiopulmonary changes, ${ }^{5}$ iatrogenic fluid overload, tocolytic therapy, ${ }^{5}$ transfusion-related acute lung injury in patients who receive blood transfusions, ${ }^{6}$ and sickle cell disease. ${ }^{7}$

Bedside imaging techniques are increasingly available to detect pulmonary pathologies, including point-of-care lung ultrasound (LUS). ${ }^{8,9}$ Lung ultrasound is a low-cost, repeatable, and

*Address correspondence to Luigi Pisani, Department of Intensive Care, Amsterdam University Medical Centers, Location AMC, Meibergdreef 9, Amsterdam 1105, The Netherlands. E-mail: luigipisani@gmail.com

†These authors contributed equally to this work. radiation-free imaging technique with a steep learning curve. It is an example of frugal innovation in critical care, thus sustainable also in resource-limited settings where conventional radiological tools are absent. ${ }^{10}$ Besides, X-ray imaging is preferably restricted in parturients because of the ionizing risk on the fetus. Lung ultrasound patterns are usually normal in parturients during the last gestational weeks, ${ }^{11}$ allowing the detection of acute pulmonary abnormalities. A recent hospital-wide study performed in Rwanda found that acute respiratory distress syndrome (ARDS) detected by LUS was a frequent and often lethal complication. ${ }^{12} \mathrm{Up}$ to one in every 10 patients with ARDS in that study was obstetric, of whom $20 \%$ died.

The frequency with which ARDS and other pulmonary complications develop and are associated with poor outcome in critically ill parturients is largely unknown in resourcelimited settings with a high maternal mortality. The objectives of the current study were to determine frequency, timing with regard to admission, type of pulmonary abnormalities detectable by LUS, and their associations with poor outcome in parturients admitted to the high dependence unit (HDU) of a large urban maternity hospital in Freetown, Sierra Leone. The primary hypothesis tested is that a large proportion of patients develop pulmonary complications identifiable by LUS and that development of these complications is associated with poor outcome. 


\section{METHODS}

Design and ethical approval. This was a prospective observational study conducted between July 2018 and February 2019 in critically ill parturients admitted to the HDU of the Princess Christian Maternity Hospital (PCMH) in Freetown, Sierra Leone. The study was approved by the Sierra Leone Ethical Research Committee on June 5, 2018. A waiver of written consent was granted because of the observational purpose of the study. The study was registered at clinicaltrials. gov (study identifier NCT 03828630).

Patients. Patients were eligible for participation if 1) parturient and 2) admitted to the HDU of the PCMH. Patients were excluded if they had passed the time window of 6 hours after admission to the HDU. Anticipated logistical reasons for exclusion were the nonavailability of the physician sonographer, for example, during weekend days, or when the dedicated physician was on call in the operating room or another ward. Patients were stratified by the presence of respiratory distress at any point during HDU stay.

Data collected. Patient clinical and ultrasound granular data were collected at predefined timepoints: on admission, after 24 hours and 48 hours, and at any point in case of patient deterioration. Demographic data collected on admission included age, weight, height, reported reason for hospital and for HDU admission, and the women gravidity, parity, and gestational age; malaria status and preexistent comorbidities; and surgical and transfusion status during the current hospital admission.

Vital signs, including heart rate, respiratory rate, temperature, neurological status, and systolic and diastolic arterial blood pressure, were captured at the moment of each LUS examination and used to compute the modified obstetric early warning score (OEWS). ${ }^{13}$ Obstetric early warning score is a composite score reflecting impairment of physiological parameters at admission. Also, peripheral pulse oximetry oxygen saturation $\left(\mathrm{SpO}_{2}\right)$, findings of chest auscultation, use of accessory muscles, and presence of nasal flaring were collected at each time point. Whenever performed for clinical reasons, point-of -care laboratory measures such as hemoglobin, glucose, and capillary lactate levels were recorded. However, no systematic laboratory examination was performed for the study purpose. Urinary output was recorded at each timepoint for catheterized patients together with the amounts of intravenous fluids administered at 24 and 48 hours. It was also recorded whether supplementary oxygen, vasopressors, and diuretics were administered. Follow-up ended at HDU discharge. Then, it was recorded whether the patients left the HDU alive or not, and status at discharge, that is, improved and discharged to ward, or transfer to tertiary hospital because of escalation of care.

Primary and secondary endpoints. The primary endpoint was the proportion of parturients with pulmonary complications detectable by LUS during stay in HDU. Secondary endpoints were the timing of appearance and types of pulmonary complications, the patients' global LUS aeration score, and occurrence of poor outcome.

The protocol for LUS. A comprehensive LUS examination was performed at each timepoint and in case of respiratory distress or clinical pulmonary edema during HDU stay (Figure 1). To minimize operator dependency and bias, LUS was performed using standardized operating procedures and structured region-based case report forms. All LUS examinations were performed by senior residents in intensive care medicine or thoracic surgery (A. d. N., M. S.), with a > 2-year experience in ultrasound procedures in critically ill patients. Dedicated bedside and remote training on at least 12 supervised examinations ${ }^{14}$ was performed together with an experienced LUS sonographer (L. P.) to familiarize with the systematic LUS scoring and case report form completion. Lung ultrasound was performed using a MyLab ${ }^{\mathrm{TM}}$ Five ultrasound machine (Esaote Spa, Genova, Italy) and a low frequency $(2.5-5 \mathrm{MHz})$ convex probe. The ultrasound machine, the probe, and the probe holder were disinfected before and after each use as from hospital indications. The patient remained in supine or semi-recumbent position, and the probe was held perpendicular to the skin.

The 12-region technique was used, in which ultrasound was performed on six areas on each side of the chest, that is, two ventral regions, two lateral regions, and two posterolateral regions, with a transversal approach to maximize lung exposition and minimize rib-related artifacts. ${ }^{15}$ The examiner scored the worst aeration pattern observed in each region using the LUS aeration score. ${ }^{16}$ Each lung field was scored from 0 to 3 as follows: "0," A-pattern with $\leq 2$ B-lines; "1," more than 2 separated B-lines; "2," multiple coalescent B-lines; or " 3 ," lung consolidation, defined as anechoic or tissue-like images arising from the pleural line that is limited in depth by an irregular border. A global LUS score was calculated at each time point and ranged from 0 to 36 . The presence of subpleural consolidations, effusion, air bronchograms, and abnormal pleural line was assessed in each field. Normal lung aeration observed using LUS was defined as all lung fields normally aerated, that is, with an A-pattern or aeration score of 0 ; bilateral interstitial syndrome was defined as the presence of two or more regions with a B-pattern (score 1 or 2) per hemithorax. ${ }^{8,17} \mathrm{~A}$ subcostal view was also acquired with the maximal and minimal diameter of the inferior vena cava, measured in motion mode $2 \mathrm{~cm}$ distal to the origin of the right atrium. ${ }^{18}$ Twelve ultrasound clips of 5 seconds each were stored after each examination on the ultrasound machine and exported as video files to a portable computer for offline analysis and quality control purposes.

Definitions. Respiratory distress was defined as the presence of one or more among the following criteria: respiratory rate $\geq 30$ breaths/minute, $\mathrm{SpO}_{2}$ /fraction of inspired oxygen $\left(\mathrm{FiO}_{2}\right) \leq 315$, and signs of difficult breathing, including the use of accessory muscles or nasal flaring.

The following definitions were used to derive the pulmonary complications from the collected clinical and ultrasound data.

Acute respiratory distress syndrome-defined according to the Kigali modification of the Berlin definition for ARDS. ${ }^{12}$ The oxygenation status was computed as the ratio between $\mathrm{SpO}_{2}$ and $\mathrm{FiO}_{2}$. Fraction of inspired oxygen was derived from the oxygen flow using the formula $\mathrm{FiO}_{2}=0.21+\left(\mathrm{O}_{2}\right.$ flow $\times$ 0.03). ${ }^{12}$ Bilateral opacities on LUS were defined as the presence of interstitial syndrome and/or two or more regions with lung consolidation bilaterally, not fully explained by effusions, lung collapse, or nodules. To fulfill the origin of edema criteria (i.e., respiratory failure not fully explained by cardiac failure or fluid overload) in a setting with absent echocardiography, all patients with a known history of 


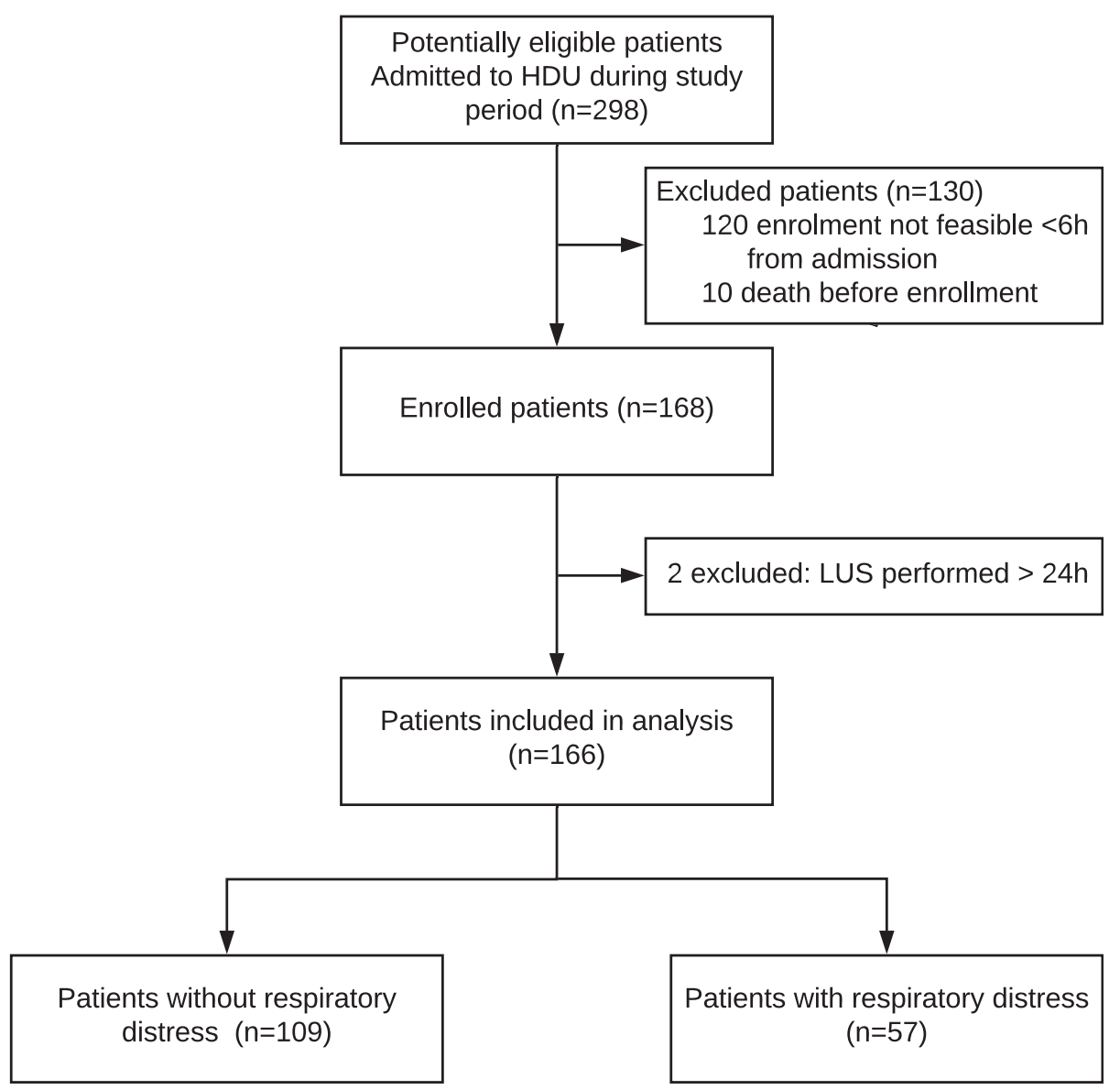

FIGURE 1. Patient flowchart. HDU = high dependency unit; LUS = lung ultrasound.

cardiac failure or diagnosed with fluid overload were excluded from this potential diagnosis.

Fluid overload or hydrostatic pulmonary edema-defined as the presence of a bilateral interstitial syndrome or pleural effusion on LUS, associated with a positive fluid balance ( $>1,000 \mathrm{~mL}$ in the last 24 hours), and/or a maximal diameter of the inferior vena cava of $>23 \mathrm{~mm}^{19}$

Pneumonia-defined as focal or multifocal interstitial syndrome and/or consolidation on LUS plus at least one of the following: temperature $>38.3^{\circ} \mathrm{C}$ or white blood cell count $>12,000 / \mathrm{mm}^{3}$ (if available).

Atelectasis-defined as the presence of mono or bilateral focal consolidations, denoting a focal loss of aeration, which did not fall in the case definitions for ARDS, pneumonia, or fluid overload.

Pleural effusion-defined as hypoechoic or anechoic collection between the parietal and visceral pleura in at least one lung region. ${ }^{17}$

Poor outcome-a composite of transfer for escalation of care or death in the HDU.

Power calculation. All patients admitted over the time span the study ran were to be included. Considering previous semester admission rates and operator availability, it was expected that more than 150 patients would be eligible during the predefined 6 months recruitment period. Considering an estimated prevalence of $20 \%$, this sample size allows to estimate the proportion of pulmonary complications with $5 \%$ precision and $95 \% \mathrm{Cl}$ in a finite population. ${ }^{20}$
Statistical analysis. Demographic, clinical, and outcome variables were presented as percentages for categorical variables and as medians with interquartile ranges for continuous variables.

Patients were stratified according to the presence or absence of respiratory distress. The proportion of patients with pulmonary complications during the HDU stay was calculated as the number of patients suffering from at least one pulmonary complication divided by the total number of patients enrolled in the study, and by the number of patients in the group with and without respiratory distress. Types of pulmonary complications and individual frequencies were reported separately for the group with and without respiratory symptoms. The chi-square statistics was used to seek significant differences across patient groups for categorical endpoints.

The Mann Whitney $U$ test and the Kruskal-Wallis test were used to compare LUS scores and other numerical variables between patients with and without respiratory symptoms on admission. Interobserver variability for the LUS scoring between the study sonographers and the expert scorer was assessed on 320 LUS images and expressed as Fleiss' kappa statistics.

A logistic regression model was used to test potential associations between occurrence of pulmonary complications and a poor outcome, defined as death in the HDU or transfer for escalation of care. The model was corrected for severity of illness on admission as estimated by the OEWS. 
All statistical analyses were performed in $R$ (version 3.3.1, www.r-project.org, R Core Team, Vienna, Austria) and graphs built using GraphPad Prism (version 7.03, www.graphpad.com, GraphPad Software, San Diego, CA). A $P$-value below 0.05 was considered significant.

\section{RESULTS}

Patient cohort. Of 298 potentially eligible patients, 166 patients were enrolled in the study. Patient flow is detailed in Figure 2. Baseline characteristics are reported in Table 1. Of all patients, 34\% presented with or developed respiratory distress during HDU stay. In total, 18 of 166 patients met the composite endpoint of a poor outcome (11\%); transfer for escalation of care occurred in $10(6 \%)$, whereas mortality was eight (5\%).

Lung ultrasound examinations and interobserver agreement. A total of 383 LUS examinations, median two (2-3) LUS/patient, were performed. All 166 patients were scanned at the day of admission, 121 also after 24 hours, and 86 also after 48 hours. Ten additional LUS examinations were performed in patients who deteriorated in between these planned examinations. Of all potential lung regions, $39(<1 \%)$ regions could not be examined because of surgical dressings or patient position. Agreement between the two study sonographers and the expert scorer in recording the LUS score for individual lung fields was kappa 0.77. Kappa statistics for anterior, lateral, and posterior lung zones were 0.76 , 0.75 , and 0.78 , respectively.

Prevalence and timing of pulmonary complications. Overall, 21\% (95\% Cl: 15-28) of patients had at least one pulmonary complication detectable by LUS during HDU stay. The incidence of pulmonary complications was higher in patients with respiratory distress versus in patients not having respiratory distress (21 of 57 [37\%] versus 14 of 109 [13\%]; $P=$ 0.001).

Timing of occurrence of pulmonary complications was similar between patients with respiratory distress $(67 \%$ on admission, and $24 \%$ and $10 \%$ in the first 24 hours or thereafter) and patients not having respiratory distress $(57 \%$ on admission, $36 \%$ and $7 \%$ in first 24 hours or thereafter).

Types of pulmonary complications and patient characteristics. Types and frequency of pulmonary complications are detailed in Table 2. Clinical characteristics are reported in Table 3. Patients with respiratory distress who had pulmonary complications detected by LUS were commonly hypoxemic and more often transferred for escalation of care. Patients with respiratory distress in the absence of pulmonary complication frequently had hemodynamic compromise in terms of higher heart rate and capillary lactates. The few patients who had a complication in the absence of respiratory distress usually had higher body mass index (BMI) and
1. Serial LUS examinations performed at h0, h24, h48 and in case of deterioration, using a 12-region protocol

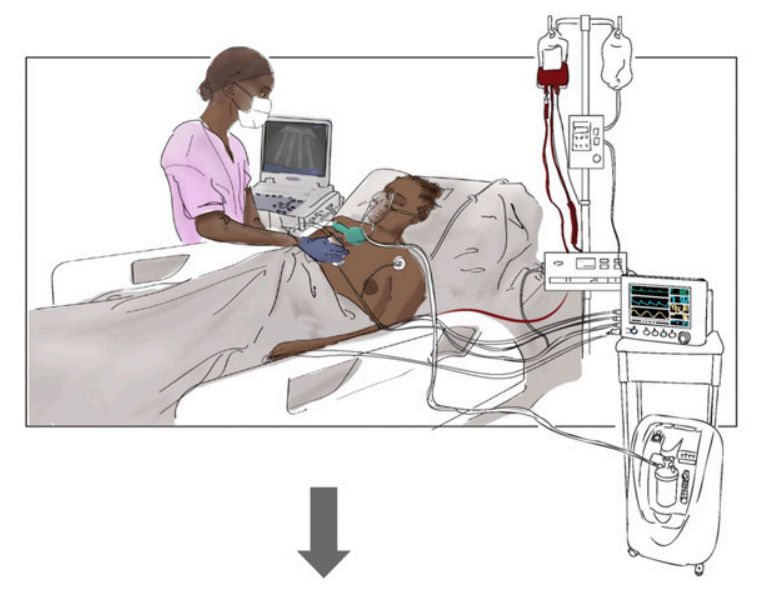

2. Each lung region was score according to the LUS score and for the presence of pleural effusion

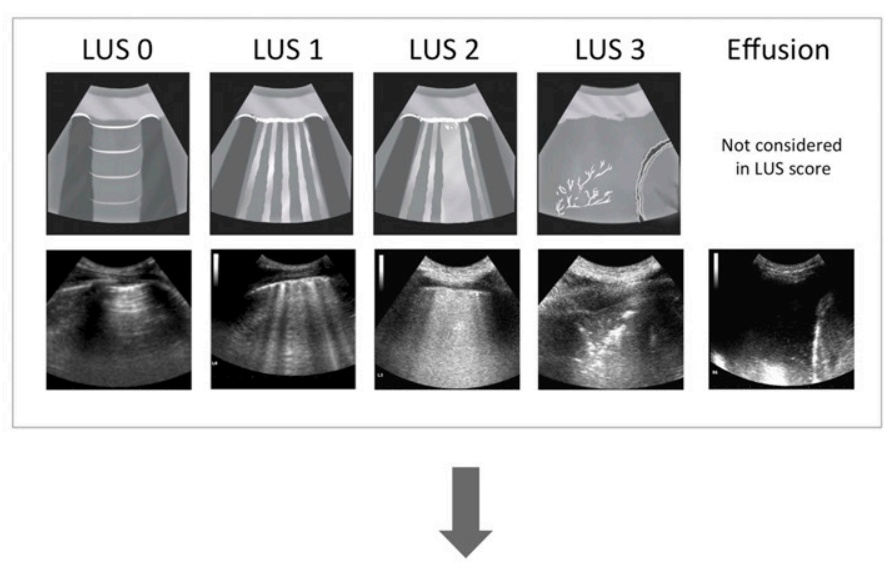

3. Case definitions were used to identify pulmonary complications. Examples are shown below:
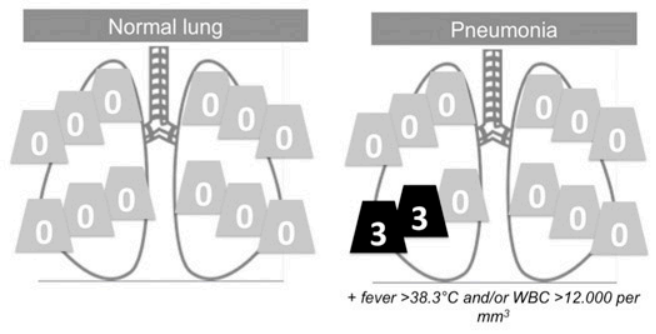

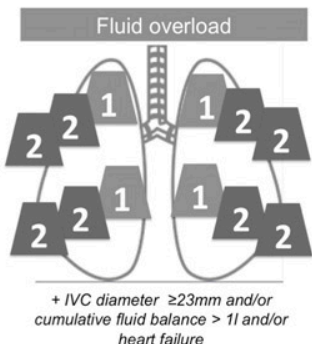

heart failure

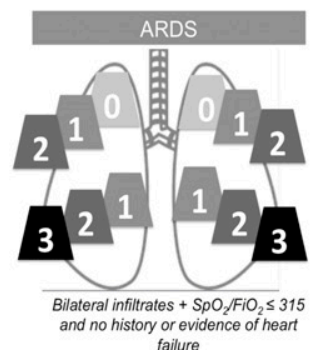

failure

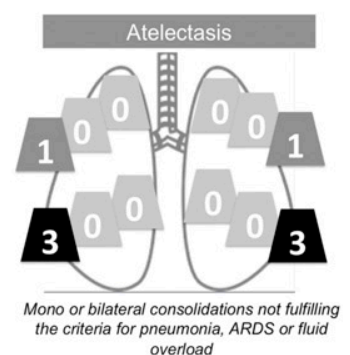

overload

FIGURE 2. Lung ultrasound protocol including the LUS score assessment and predefined case definitions of pulmonary complications integrating LUS and clinical data. One example for each pulmonary complication case definition is shown. Lung ultrasound images were drawn from the study saved clips, with additional details in the text. $\mathrm{FiO}_{2}=$ fraction of inspired oxygen; IVC = inferior vena cava; $\mathrm{LUS}=$ lung ultrasound; $\mathrm{SpO}{ }_{2}=$ peripheral pulse oximetry oxygen saturation. This figure appears in color at www.ajtmh.org. 
TABLE 1

Patient characteristics at baseline

\begin{tabular}{|c|c|}
\hline Variable & Number \\
\hline \multicolumn{2}{|l|}{ General epidemiology } \\
\hline Age (years) & $25(22,30)$ \\
\hline Gestation age (weeks) & $36(32,38)$ \\
\hline Gravidity & $3(2,4)$ \\
\hline Parity & $1(0,3)$ \\
\hline Body mass index $\left(\mathrm{kg} / \mathrm{m}^{2}\right)$ & $23.9(22.2,25.7)$ \\
\hline \multicolumn{2}{|l|}{ Clinical features } \\
\hline Obstetric early warning score total score & $3(2,4)$ \\
\hline Altered sensorium, $n(\%)^{\star}$ & $16(9.6)$ \\
\hline Mean arterial blood pressure $(\mathrm{mmHg}) \dagger$ & $95(82,110)$ \\
\hline Heart rate (beats/minute) $\ddagger$ & $105(95,120)$ \\
\hline \multicolumn{2}{|l|}{ Respiratory features } \\
\hline Respiratory rate (movements/minute) & $24(22,28)$ \\
\hline $\mathrm{SpO}_{2}$ & $99(98,99)$ \\
\hline $\mathrm{SpO}_{2} /$ fraction of inspired oxygen & $467(462,471)$ \\
\hline Oxygen therapy, yes, $n(\%)$ & $29(17.4)$ \\
\hline \multicolumn{2}{|l|}{ Biology } \\
\hline Hemoglobin $(g / d L) \S$ & $7.9(6.3,9.8)$ \\
\hline Capillary lactates levels $(\mathrm{mmol} / \mathrm{L}) \|$ & $4.5(2.6,7.1)$ \\
\hline Positive to malaria, $n(\%)$ & $12(7.2)$ \\
\hline \multicolumn{2}{|l|}{ Reason of admission, $n(\%)$} \\
\hline Antepartum hemorrhage & $45(28.7)$ \\
\hline Postpartum hemorrhage & $14(8.9)$ \\
\hline Uterine rupture & $22(14)$ \\
\hline Severe preeclampsia & 30 (19.1) \\
\hline Obstructed labor & $15(9.6)$ \\
\hline Ectopic pregnancy & $7(4.5)$ \\
\hline Sepsis & $11(7.0)$ \\
\hline Sickle cell disease & $5(3.2)$ \\
\hline \multicolumn{2}{|l|}{ Type of delivery/surgery, $n$ (\%) } \\
\hline Spontaneous vaginal delivery & $17(10.8)$ \\
\hline Cesarean section & $68(43.3)$ \\
\hline Surgery (other than cesarean) & 37 (23.6) \\
\hline \multicolumn{2}{|l|}{ Type of anesthesia, $n(\%)$} \\
\hline General anesthesia & $84(53.5)$ \\
\hline Spinal anesthesia & $18(11.5)$ \\
\hline \multicolumn{2}{|c|}{$\begin{array}{l}\mathrm{SpO}_{2}=\text { peripheral pulse oximetry oxygen saturation. Data are presented as median } \\
\text { (interquartile range) or proportion (\%). } \\
\text { * Patients only responsive to painful stimulus or unresponsive on admission. } \\
\text { † Missing in three patients. } \\
\text { † Missing in one patients. } \\
\S \text { Missing in five patients. } \\
\| \text { Missing in } 46 \text { patients. }\end{array}$} \\
\hline
\end{tabular}

suffered from atelectasis. Most patients never developed respiratory distress and had negative LUS examinations.

Lung ultrasound score. Overall, LUS examinations in which a pulmonary complication was detected had higher global LUS score than negative LUS examinations (four [3-9] versus zero [0-1]; $P<0.001)$. The baseline global LUS score was different in the four patient groups, and for different pulmonary complications (Figure 3).

Association with outcome. The occurrence of a poor outcome was highest in patients with respiratory distress and pulmonary complications detected by LUS (Table 2). The occurrence of one or more pulmonary complication was associated with poor outcome (odds ratio: 5.0; 95\% Cl: $1.7-14.6 ; P=0.003)$.

\section{DISCUSSION}

In this study of critically ill obstetric patients in a resourcelimited HDU in Africa, pulmonary complications detectable by LUS were frequent, affecting one of five patients. Most of these pulmonary complications had an early onset. Acute respiratory distress syndrome and fluid overload occurred with equal prevalence. Presence or development of pulmonary complications diagnosed by LUS was associated with increased risk of poorer outcome.

Strengths of this study include the use of clear case definitions integrating clinical parameters with LUS variables. The prospective design and serial LUS follow-up allowed capturing complications that were not present on admission. Examinations were performed using an abdominal ultrasound probe that is widely available in obstetric units. The stratification by respiratory distress allowed investigation of the clinical meaning of LUS findings in critically ill parturients in a setting where resources are extremely limited.

Patients with abnormal lung findings identified through LUS and respiratory distress frequently had ARDS. The period prevalence of ARDS mirrors previous findings in a hospitalwide study in Rwanda. ${ }^{12}$ Whereas the absence of blood gas analysis and chest radiography did not allow to verify the diagnosis of ARDS against the current Berlin definition for ARDS, ${ }^{21}$ the used criteria were individually validated. ${ }^{12,22}$ Patients were exposed to ARDS risk factors such as sepsis, surgery, malaria, and whole blood transfusions, ${ }^{23,24}$ whereas pneumonia was rare.

Pulmonary edema was another diagnosis in patients with pulmonary complications and respiratory symptoms. The incidence of pulmonary edema in the current cohort is in line with estimates ranging from $0.1 \%$ in normal pregnancy to $10 \%$ in patients with preeclampsia. ${ }^{25}$ Physiologic changes of

TABLE 2

Types of pulmonary complications detected by LUS in patients with and without respiratory distress, and associated patients' outcomes

\begin{tabular}{|c|c|c|c|c|c|}
\hline & \multicolumn{2}{|c|}{ Respiratory distress $(n=57)$} & \multicolumn{2}{|c|}{ No Respiratory distress $(n=109)$} & \multirow[b]{2}{*}{$P$-value } \\
\hline & With LUS complication $(n=21)$ & No LUS complication $(n=36)$ & With LUS complication $(n=14)$ & No LUS complication ( $n=95)$ & \\
\hline Pulmonary complications, ${ }^{\star} n$ (\%) & & & & & $<0.001$ \\
\hline Acute respiratory distress syndrome & $6(28.6)$ & - & $0(0)$ & - & \\
\hline Fluid overload & $6(28.6)$ & - & $0(0)$ & - & \\
\hline Effusion & $8(38.1)$ & - & $3(21.4)$ & - & \\
\hline Pneumonia & $2(9.5)$ & _- & $1(7.1)$ & - & \\
\hline Atelectasis & $6(28.6)$ & - & $11(78.6)$ & - & \\
\hline \multicolumn{6}{|l|}{ Outcomes } \\
\hline Length of stay (days) & $3.0(2.0,3.2)$ & $3.0(2.0,4.2)$ & $3.5(3.0,4.0)$ & $3.0(2.0,4.0)$ & 0.541 \\
\hline Poor outcome, $n(\%)$ & $6(28.6)$ & $5(13.9)$ & $3(21.4)$ & $4(4.2)$ & 0.004 \\
\hline Death, $n(\%)$ & $0(0)$ & $5(13.9)$ & $1(7.1)$ & $2(2.1)$ & - \\
\hline Transfer, $n(\%)$ & $6(28.6)$ & $0(0)$ & $2(14.3)$ & $2(2.1)$ & _ \\
\hline
\end{tabular}

LUS = lung ultrasound. Data are presented as median (interquartile range) or proportion (\%).

${ }^{\star}$ Nonexclusive categories. 
TABLE 3

Patient characteristics in the different study groups

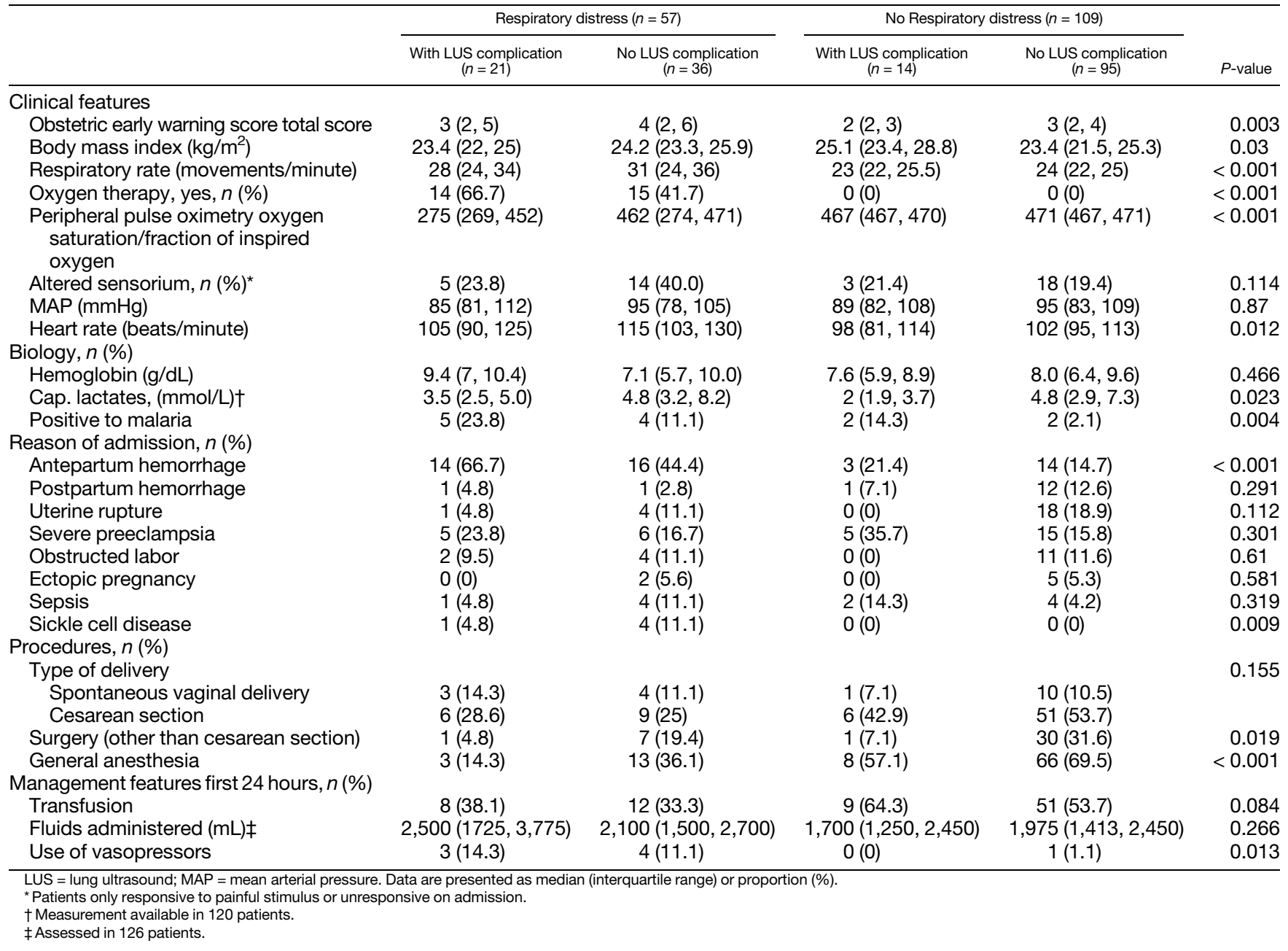

pregnancy, preeclampsia, and puerperal cardiomyopathy predispose parturients to pulmonary oedema. ${ }^{5}$ The combination of nifedipine use and magnesium sulfate, acute kidney injury related to severe preeclampsia and iatrogenic fluid overload may have contributed to pulmonary congestion. ${ }^{26}$

Lung ultrasound excluded a pulmonary complication in more than half of patients with respiratory distress. Lung ultrasound high negative predictive value is supported by findings in previous studies and allows to swiftly exclude parenchymal involvement in the dyspneic patient. ${ }^{8,27}$ These patients likely had other reasons to develop respiratory symptoms, such as metabolic acidosis and hemorrhagic anemia. Malaria may also induce compensatory tachypnea and represents a risk factor for lung injury. ${ }^{28}$ The exclusion of a pulmonary complication in dyspneic parturients was a key finding of this study and may provide the most added value in daily clinical practice in the obstetric population, which generally has a low pretest probability of pulmonary findings. Respiratory distress in combination with a negative LUS may also suggest a pulmonary embolism, which could have been captured with compression ultrasonography. ${ }^{29}$ However, to keep burden of the study acceptable, compression ultrasonography was not part of the study protocol.
Patients with a positive LUS in the absence of respiratory symptoms did not require oxygen and had normal oxygenation. Previous studies documented how diffuse B-lines may be present in asymptomatic parturients. ${ }^{11,30}$ The finding of frequent atelectasis in this group may be explained by the exposure to risk factors such as higher BMI and general anesthesia. ${ }^{27,31}$

Pulmonary complications were significantly associated with poorer outcome, confirming findings in non-obstetric patients in Africa ${ }^{12}$ and Asia. $^{32}$ This does not imply a causal relationship with mortality, as parturients largely die of direct obstetric complications such as hemorrhage, eclampsia, and sepsis. $^{3,33}$ Yet, pulmonary complications led to frequent escalation of care. Although most obstetric critical illness can be treated in medium care units, ${ }^{3,34}$ intensive care unit beds and mechanical ventilators are scarcely available in Africa. ${ }^{3,35}$ Identifying patients with complications may help the caregivers to allocate the scarce monitoring, oxygen, and ventilation resources.

This study faces limitations. A considerable number of patients were missed as died before recruitment or when it was not possible to perform LUS within 6 hours from admission because of logistical reasons. However, this strict predefined inclusion window was designed to pinpoint 
A

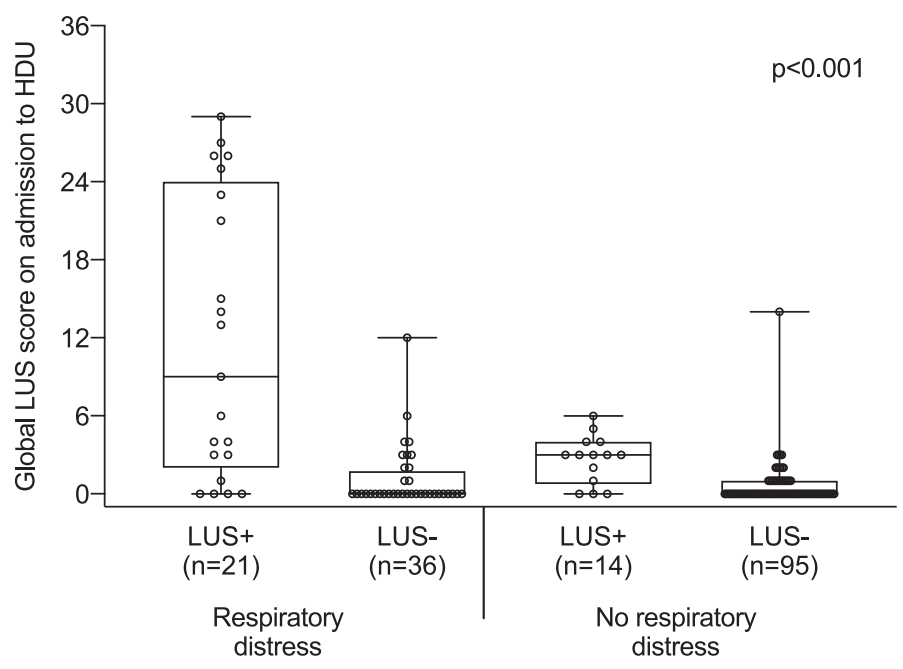

B

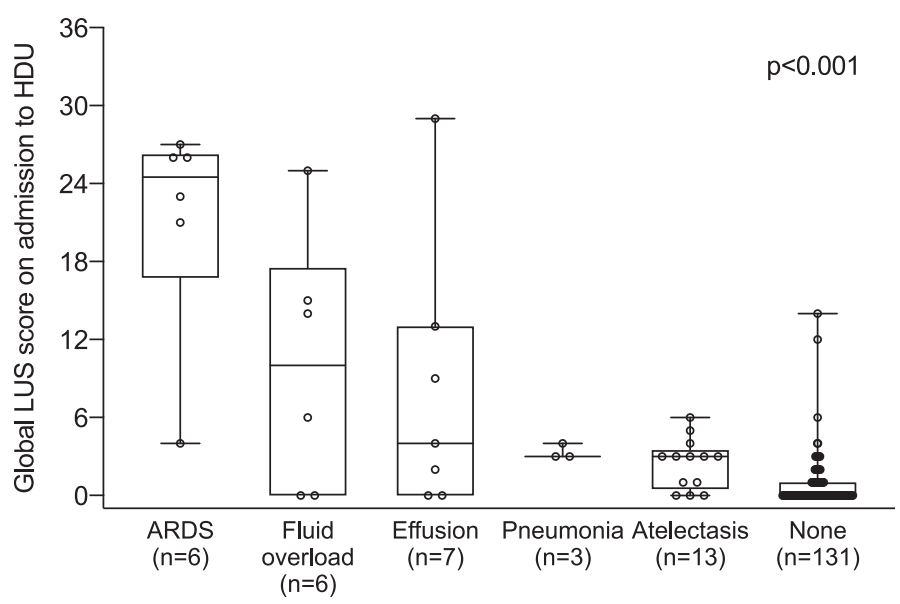

C

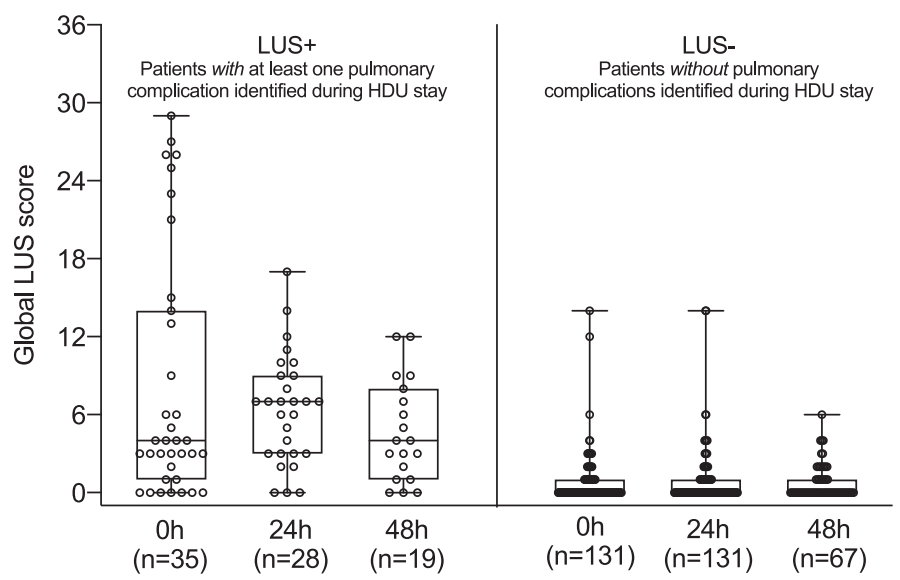

FIGURE 3. Baseline global lung ultrasound score on patient admission expressing loss of lung aeration in the different patient groups (A) and across different pulmonary complications (B). Individual global lung ultrasound scores are also represented at admission, at 24 hours and 48 hours (C). The middle line represents the median, the lower hinge represents the first quartile, the upper hinge represents the third quartile, and the whiskers extend to the minimum and maximum values. ARDS = acute respiratory distress syndrome; LUS = lung ultrasound.

pulmonary conditions observed early in the course of obstetric critical illness. Pulmonary case definitions were defined from granular data at study completion. Although this minimizes observation bias, it may lead to under or overestimation of complications. No other radiological imaging techniques were available in this setting to confirm the LUS findings. This limitation is mitigated by the body of literature validating LUS against reference methods for the conditions 
investigated..$^{8,31,36}$ Echocardiography was not available to definitively exclude a peripartum cardiomyopathy; thus, patients with a history of cardiac failure were excluded a priori from the diagnosis of ARDS. Similarly, compression ultrasonography for the exclusion of deep vein thrombosis was not performed. Finally, the analysis regarding impact of pulmonary complications was limited by the unknown survival status of transferred patients.

\section{CONCLUSION}

In this cohort of critically ill obstetric patients in a resourcelimited HDU, LUS was a useful and safe imaging tool to identify or exclude pulmonary involvement in patients with or without respiratory distress. Pulmonary complications occurred early, and their presence or development was associated with poor outcome.

Received August 11, 2020. Accepted for publication September 27, 2020.

Published online December 14, 2020.

Acknowledgments: We are deeply grateful to the HDU nursing staff who assisted in the daily examinations and study conduct. We are also grateful to PCMH leadership and all treating teams for the constant collaboration.

Financial support: This study was funded by the Amsterdam University Medical Centers, location AMC and Doctors with Africa-CUAMM, Padova, Italy.

Disclosure: Individual participant data collected during the study will be available after de-identification, immediately following publication, with no end date. Data will be available to researchers providing a methodologically sound proposal. Proposals should be directed to the corresponding author.

Authors' addresses: Luigi Pisani, Department of Intensive Care, Amsterdam University Medical Centers-Location AMC, Amsterdam, The Netherlands, and Mahidol Oxford Tropical Medicine Research Unit, Bangkok, Thailand, E-mail: luigipisani@gmail.com. Anna De Nicolo and Salvatore Grasso, Intensive Care Unit, Department of Emergency and Organ Transplantation (DETO), University of Bari, Bari, Italy, E-mails: annuu@hotmail.it and salvatore.grasso@uniba.it. Marcella Schiavone, Angela De Palma, and Giuseppe Marulli, Thoracic surgery Unit, University of Bari, Bari, Italy, E-mails: marcella.schiavone@gmail.com, angela.depalma@uniba.it, and giuseppe.marulli@uniba.it. Adetunji O. Adeniji, Edward Ejiro Emuveyan, Alimamy P. Koroma, and Enzo Pisani, Department of Obstetrics and Gynecology, Princess Christian Maternity Hospital, Freetown, Sierra Leone, E-mails: tunji1802@yahoo.com, edwardemuveyan@ yahoo.com, apkoroma2@yahoo.co.uk, and e.pisani@cuamm.org. Francesco Di Gennaro, Department of Infectious Diseases, University of Bari, Bari, Italy, E-mail: cicciodigennaro@yahoo.it. Patricia C. Henwood, Department of Emergency Medicine, Brigham and Women's Hospital, Boston, MA, and Department of Emergency Medicine, Thomas Jefferson University Hospital, Philadelphia, PA, E-mail: trish.henwood@gmail.com. Stije Leopold, Mahidol Oxford Research Unit, Mahidol University, Bangkok, Thailand, E-mail: stijeleopold@ gmail.com. Claudia Marotta and Giovanni Putoto, Operational research Unit, Doctors with Africa CUAMM, Padua, Italy, E-mails: marotta.claudia@gmail.com and g.putoto@cuamm.org. James Russel, Department of Cardiology, Connaught Hospital, Freetown, Sierra Leone, E-mail: jamesbwrussell@gmail.com. Ary Serpa Neto, Critical Care Medicine, Hospital Israelita Albert Einstein, Sao Paulo, Brazil, E-mail: ary.neto2@einstein.br. Arjen M. Dondorp, Faculty of Tropical Medicine, Mahidol-Oxford Tropical Medicine Research Unit (MORU), Bangkok, Thailand, E-mail: arjen@tropmedres.ac. Eva Hanciles, Univerity of Sierra Leone, Freetown, Sierra Leone, E-mail: ehanchris@ yahoo.co.uk. Michael M. Koroma, Department of Anesthesiology, Princess Christian Maternity Hospital, Freetown, Sierra Leone, E-mail: drmichaelkoroma@gmail.com. Marcus J. Schultz, Mahidol Oxford
Research Unit, Mahidol University, Bangkok, Thailand, and Department of Intensive Care, Amsterdam University Medical Centers-Location AMC, Amsterdam, The Netherlands, E-mail: marcus.j.schultz@gmail.com.

This is an open-access article distributed under the terms of the Creative Commons Attribution (CC-BY) License, which permits unrestricted use, distribution, and reproduction in any medium, provided the original author and source are credited.

\section{REFERENCES}

1. CIA, 2018. The World Factbook. Available at: https://www.cia.gov/ library/publications/the-world-factbook/. Accessed February 8, 2018.

2. Adhikari NKJ, Fowler RA, Bhagwanjee S, Rubenfeld GD, 2010. Critical care and the global burden of critical illness in adults. Lancet 376: 1339-1346.

3. Zeeman GG, 2006. Obstetric critical care: a blueprint for improved outcomes. Crit Care Med 34: S208-S214.

4. Neligan PJ, Laffey JG, 2011. Clinical review: special populations critical illness and pregnancy. Crit Care 15: 227.

5. Dennis AT, Solnordal CB, 2012. Acute pulmonary oedema in pregnant women. Anaesthesia 67: 646-659.

6. Michala L, Madhavan B, Win N, De Lord C, Brown R, 2008. Transfusion-related acute lung injury (TRALI) in an obstetric patient. Int J Obstet Anesth 17: 66-69.

7. Yawn BP, John-Sowah J, 2015. Management of sickle cell disease: recommendations from the 2014 expert panel report. Am Fam Phys 92: 1069-1076.

8. Volpicelli $G$ et al., 2012. International evidence-based recommendations for point-of-care lung ultrasound. Intensive Care Med 38: 577-591.

9. Lichtenstein DA, Mezière GA, 2008. Relevance of lung ultrasound in the diagnosis of acute respiratory failure the BLUE protocol. Chest 134: 117-125.

10. Mekontso Dessap A, 2018. Frugal innovation for critical care. Intensive Care Med 45: 252-254.

11. Arbeid E, Demi A, Brogi E, Gori E, Giusto T, Soldati G, Vetrugno L, Giunta F, Forfori F, 2017. Lung ultrasound pattern is normal during the last gestational weeks: an observational pilot study. Gynecol Obstet Invest 82: 398-403.

12. Riviello ED, Kiviri W, Twagirumugabe T, Mueller A, BannerGoodspeed VM, Officer L, Novack V, Mutumwinka M, Talmor DS, Fowler RA, 2016. Hospital incidence and outcomes of the acute respiratory distress syndrome using the Kigali modification of the Berlin definition. Am J Respir Crit Care Med 193: 52-59.

13. Singh S, McGlennan A, England A, Simons R, 2012. A validation study of the $\mathrm{CEMACH}$ recommended modified early obstetric warning system (MEOWS). Anaesthesia 67: 12-18.

14. See KC, Ong V, Wong SH, Leanda R, Santos J, Taculod J, Phua J, Teoh CM, 2016. Lung ultrasound training: curriculum implementation and learning trajectory among respiratory therapists. Intensive Care Med 42: 63-71.

15. Bouhemad B, Zhang M, Lu Q, Rouby JJ, 2007. Clinical review: bedside lung ultrasound in critical care practice. Crit Care 11: 205.

16. Bouhemad B, Brisson H, Le-Guen M, Arbelot C, Lu Q, Rouby JJ, 2011. Bedside ultrasound assessment of positive endexpiratory pressure-induced lung recruitment. Am J Respir Crit Care Med 183: 341-347.

17. KruisselbrinkR, Chan V, Cibinel GA, Abrahamson S, Goffi A, 2017. I-AIM (indication, acquisition, interpretation, medical decisionmaking) framework for point of care lung ultrasound. Anesthesiology 127: 568-582.

18. Weekes AJ, Tassone HM, Babcock A, Quirke DP, Norton HJ, Jayarama K, Tayal VS, 2011. Comparison of serial qualitative and quantitative assessments of caval index and left ventricular systolic function during early fluid resuscitation of hypotensive emergency department patients. Acad Emerg Med 18: 912-921.

19. Lee CWC, Kory PD, Arntfield RT, 2016. Development of a fluid resuscitation protocol using inferior vena cava and lung ultrasound. J Crit Care 31: 96-100.

20. Sergeant, 2019. Epitools. Epitools Epidemiological CalculatorsAusvet. Available at: http://epitools.ausvet.com.au. Accessed July 2019. 
21. ARDSDefinition Task Force: Ranieri VM, Rubenfeld GD, Thompson BT, Ferguson ND, Caldwell E, Fan E, Camporota L, Slutsky AS, 2012. Acute respiratory distress syndrome. JAMA 307: 2526-2533.

22. Vercesi V et al., 2018. External confirmation and exploration of the Kigali modification for diagnosing moderate or severe ARDS. Intensive Care Med 44: 523-524.

23. Mac Sweeney R, McAuley DF, 2016. Acute respiratory distress syndrome. Lancet 388: 2416-2430.

24. Bandi VD, Munnur U, Matthay MA, 2004. Acute lung injury and acute respiratory distress syndrome in pregnancy. Crit Care Clin 20: 577-607.

25. Hammad $Y$, Hasanin A, Elsakka A, Refaie A, Abdelfattah D, Rahman SA, Zayed M, Hassabelnaby Y, Mukhtar A, Omran A, 2019. Thoracic fluid content: a novel parameter for detection of pulmonary edema in parturients with preeclampsia. J Clin Monit Comput 33: 413-418.

26. Xiao C, Gangal M, Abehhaim H, 2014. Effect of magnesium sulfate and nifedipine on the risk of developing pulmonary edema in preterm births. J Perinat Med 42: 585-589.

27. Ashton-Cleary DT, 2013. Is thoracic ultrasound a viable alternative to conventional imaging in the critical care setting? $\mathrm{Br} J$ Anaesth 111: 152-160.

28. Taylor WRJ, Hanson J, Turner GDH, White NJ, Dondorp AM, 2012. Respiratory manifestations of malaria. Chest 142: 492-505.

29. Lichtenstein DA, 2015. BLUE-protocol and FALLS-protocol: two applications of lung ultrasound in the critically ill. Chest 47: 1659-1670.
30. Krawczyk P, Jastrzębska A, Sałapa K, Szczeklik W, Andres J, 2019. Abnormal lung ultrasound pattern during labor: a prospective cohort pilot study. J Clin Ultrasound 47: 261-266.

31. Monastesse A, Girard F, Massicotte N, Chartrand-Lefebvre C, Girard M, 2017. Lung ultrasonography for the assessment of perioperative atelectasis: a pilot feasibility study. Anesth Analg 124: 494-504.

32. Leopold SJ et al., 2018. Point-of-care lung ultrasound for the detection of pulmonary manifestations of malaria and sepsis: an observational study. PLoS One 13: e0204832.

33. Marotta C et al., 2020. Epidemiology, outcomes, and risk factors for mortality in critically ill women admitted to an obstetric highdependency unit in Sierra Leone. Am J Trop Med Hyg. doi: 10.4269/ajtmh.20-0623.

34. Marotta C et al., 2020. Cost-utility of intermediate obstetric critical care in a resource-limited setting: a value-based analysis. Ann Glob Heal 86: 82.

35. Baelani I, Jochberger S, Laimer T, Otieno D, Kabutu J, Wilson I, Baker T, Dünser MW, 2011. Availability of critical care resources to treat patients with severe sepsis or septic shock in Africa: a self-reported, continent-wide survey of anaesthesia providers. Crit Care 15: R10.

36. Mojoli F, Bouhemad B, Mongodi S, Lichtenstein D, 2019. Lung ultrasound for critically ill patients. Am J Respir Crit Care Med 199: 701-714. 